\title{
Meningeoma frontal numa paciente com síndrome depressiva maior crônica
} Frontal Meningeoma in a Patient with Chronic Major Depression

\author{
César Ricardo Skaf ${ }^{1}$, Airton Yamada ${ }^{2}$, Sérgio Akamine ${ }^{3}$ e Geraldo Busatto Filho ${ }^{4}$
}

\section{Introdução}

Sintomas psiquiátricos estão presentes com freqüência em transtornos neurológicos como acidentes vasculares cerebrais (AVCs), tumores cerebrais, doença de Parkinson, coréia de Huntington e epilepsias, ${ }^{1,2}$ e muitas vezes surgem como primeira manifestação da doença neurológica. ${ }^{3}$ Essas manifestações psiquiátricas podem ocorrer sob a forma de síndromes depressivas, ${ }^{4}$ sendo também comuns alterações cognitivas, mudanças de personalidade e sintomas psicóticos. ${ }^{1,5}$ Em conseqüência, doenças cerebrais orgânicas são um diagnóstico diferencial a ser considerado em pacientes que apresentam aparente quadro psiquiátrico primário. Em classificações atuais, como a $4^{\mathrm{a}}$ edição do Manual Diagnóstico e Estatístico de Transtornos Mentais da Associação Psiquiátrica Americana (Diagnostic and Statistical Manual of Mental Disorders - DSMIV), o diagnóstico de transtornos mentais maiores só pode ser firmado se excluídas causas orgânicas; na presença destas, o diagnóstico passa a ser o de transtorno mental secundário a condição médica geral. ${ }^{6}$

Atualmente, técnicas sofisticadas e cada vez mais acessíveis de neuroimagem, como tomografia computadorizada (TC), ressonância magnética $(\mathrm{RM})$ e tomografia por emissão de fóton único (single photon emission computed tomography - SPECT) podem ser utilizadas como recurso complementar na prática psiquiátrica, em casos selecionados nos quais dados de história clínica, exames psíquico, físico e/ou neurológico trazem alguma suspeita da presença de condição médica geral subjacente. ${ }^{7}$ Apresentamos aqui o caso de uma paciente de meia idade que sofria de quadro psiquiátrico crônico, com predominância de sintomas depressivos de início insidioso e piora progressiva, e que através de avaliação por exames de neuroimagem, teve diagnosticado um tumor (meningeoma) de grandes proporções localizado em região frontal.

\section{Apresentação do Caso}

Paciente S.M.T.S., 43 anos, sexo feminino, confeiteira, católica, foi trazida a um ambulatório de Psiquiatria Geral com histó- ria crônica de tristeza, falta de ânimo e energia para desempenhar quaisquer tarefas, pessimismo em relação a todos os eventos de seu cotidiano e ao futuro, e isolamento social. Este quadro se iniciara sete anos antes, com sintomas discretos, poucos meses após a morte do marido num acidente automobilístico, e vinha evoluindo com piora lenta e progressiva, sem remissões. Observava-se também retardo psicomotor, falta de apetite com emagrecimento moderado, insônia tanto inicial como terminal, com hipersônia durante o dia. Dois anos após o início do quadro, a paciente abandonou suas atividades profissionais, passando a ter também dificuldades cada vez maiores em tarefas domésticas. Recentemente, a sensação de tristeza, inutilidade, falta de energia e fadiga vinham sendo experimentadas todos os dias, e a paciente chegava a dormir ininterruptamente por 12 horas num dia. Queixava-se também de falta de concentração e memória, dificuldades para pensar e tomar decisões, e considerava freqüentemente que morrer seria a solução para seus problemas. Nos três meses que antecederam a consulta, desenvolveu quadro de alucinações visuais freqüentes e nítidas, vendo uma figura humana feminina bem formada que trazia a ela perplexidade e dúvida. No último mês, vinha também escutando ruídos e palavras pouco claras. Ajuda médica não havia sido procurada antes, em parte pelo fato de a família entender os sintomas da paciente como preguiça e dificuldade em querer se ajudar. Três das suas quatro filhas haviam saído de casa pelas dificuldades em conviver com a mãe tão desanimada e, recentemente, a paciente passava grande parte do dia sozinha. A decisão familiar de procurar ajuda médica foi tomada quando a paciente entrou em isolamento quase completo, praticamente já não saindo da cama.

A paciente não apresentava antecedentes pessoais psiquiátricos incluindo episódios prévios de mania ou hipomania, e tinha história familiar negativa para transtornos psiquiátricos maiores. Negava também antecedentes pessoais ou sintomas físicos sugestivos de doença física geral ou neurológica, à exceção de cefaléias frontais discretas e esporádicas.

Não foram observadas alterações de exame físico geral na ava-

1. Pós-graduando do Departamento de Psiquiatria da Faculdade de Medicina da Universidade de São Paulo.

2. Chefe do Centro de Medicina Nuclear do Paraná (Cermen).

3. Médico Radiologista do Hospital Vita (Curitiba).

4. Pesquisador do Departamento de Psiquiatria da Faculdade de Medicina da Universidade de São Paulo.

Rev Bras Psiquiatr, 21 (2), 1999 
liação inicial. No exame neurológico, detectou-se tremor fino de mãos e redução de força muscular em membros, além de discreta palidez em exame de fundo de olho à esquerda. No exame psíquico, chamavam a atenção o humor polarizado para depressão, lentificação do curso do pensamento e da linguagem. Não apresentava, no exame psíquico, comportamento alucinatório. Mostrava em alguns momentos dificuldade de focar a atenção aos estímulos da entrevista, alguma hipomnésia para eventos recentes, e desorientação temporal flutuante e discreta, percebida apenas no exame mais rigoroso. Com base nos critérios do DSM-IV, ${ }^{6}$ foram levantadas as hipóteses de transtorno depressivo maior crônico, grave, com aspectos psicóticos, ou transtorno mental secundário à condição médica geral.

Exames laboratoriais gerais, de líquor, eletrocardiograma e raio-X de tórax foram normais. No eletroencefalograma, detectou-se ritmo de base discreta e difusamente lento. A paciente foi submetida a exame de RM de crânio, que mostrou volumosa lesão expansiva de contornos bosselados, localizada em região frontal bilateralmente com predomínio à direita, com edema e compressão intensa de fossa anterior, e impregnação homogênea após administração de contraste paramagnético, em quadro sugestivo de meningeoma (figura 1).

Figura 1 - Exame de ressonância magnética estrutural
A

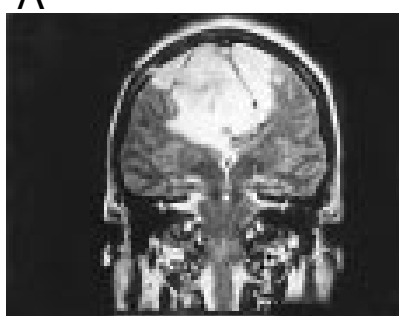

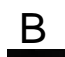

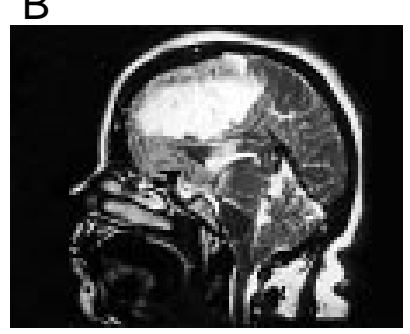

Imagens pesadas em T2 de ressonância magnética do crânio (TR 3116, TE 130), obtidas após administração venosa de contraste paramagnético (A-corte coronal; B-corte sagital). Notase a presença de volumosa lesão expansiva bilateral em região frontal, com intensa impregnação homogênea pelo contraste, sugestiva de meningeoma.

Foi realizado também exame de fluxo sanguíneo cerebral regional (FSCr) através de SPECT, que mostrou redução dramática e extensa de FSCr fronto-parietal bilateralmente, que se estendia para além dos limites do tumor identificado à ressonância magnética (figura 2).

Após diagnosticada a lesão cerebral a paciente foi encaminhada para um serviço de oncologia, onde decidiu-se por intervenção cirúrgica. Durante o período de oito semanas que transcorreu até a data da cirurgia, a paciente recebeu amitriptilina em doses de até $75 \mathrm{mg} / \mathrm{dia}$, com alguma melhora dos sintomas psiquiátricos. A cirurgia da paciente foi malsucedida, com sangramento cerebral extenso no período pós-operatório imediato, que progrediu para coma e óbito em 30 horas. O exame histológico da peça cirúrgica confirmou o diagnóstico de meningeoma.
Figura 2 - Exame de SPECT cerebral

A

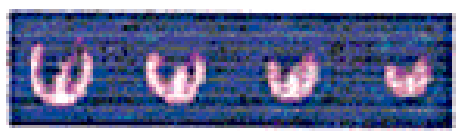

B

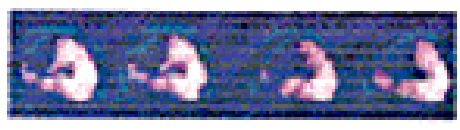

C

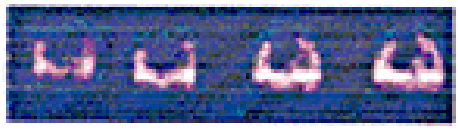

Imagens de fluxo sanguíneo cerebral regional (FSCr) obtidas através de tomografia computadorizada por emissão de fótons (SPECT) após administração venosa do traçador ${ }^{99 \mathrm{~m}} \mathrm{Tc}-$ dímero de etilcisteinato ( $\left.{ }^{99 \mathrm{~m}} \mathrm{Tc}-\mathrm{ECD}\right)$ (A-cortes axiais, B-cortes sagitais; C-cortes coronais). Nota-se ausência dramática de captação do traçador predominantemente em córtex frontal bilateralmente, de extensão mais ampla que os limites do tumor identificado à ressonância magnética (figura 1).

\section{Discussão}

A paciente aqui apresentada sofria de um tumor cerebral extenso que foi detectado através de exame de RM de crâneo, após vários anos de história psiquiátrica com sintomas depressivos marcantes e alterações cognitivas discretas, de curso insidioso e piora progressiva, e com a adição recente de sintomas alucinatórios.

O caso ilustra a bem conhecida relação entre sintomas mentais e lesões tumorais expansivas no cérebro. Sintomas psiquiátricos têm sido documentados em casos de tumores cerebrais, em alguns casos precedendo o diagnóstico da doença orgânica. ${ }^{8}$ Entre as manifestações psiquiátricas descritas, síndromes de depressão e/ou apatia são relatadas em 17\% a 50\% dos indivíduos com tumor cerebral. ${ }^{9}$ Depois dos astrocitomas, os meningeomas são os tumores cerebrais mais freqüentes nas comunidades ocidentais, representando $15 \%$ a $20 \%$ dos mesmos. ${ }^{10}$ Ocorrem com mais freqüência em mulheres (60\%), com pico de incidência ao redor dos 45 anos. ${ }^{10}$ Os meningeomas tendem a crescer lenta e arrastadamente ao longo de anos, podendo ser assintomáticos e aparecer somente como achado "post mortem". Em particular, meningeomas frontais podem alcançar dimensões gigantes antes de produzirem sintomas neurológicos que permitam o diagnóstico. ${ }^{2}$

No presente caso, pode-se levantar a hipótese de que a apresentação psicopatológica do quadro da paciente, com predomínio de sintomas depressivos crônicos, estava relacionada à localização frontal do tumor cerebral. Há diversas descrições de associações específicas entre quadros depressivos e lesões frontais bem definidas. ${ }^{4}$ Estudos de populações com história prévia de AVC mostram um excesso de síndromes depressivas associadas a lesões em áreas do córtex pré-frontal esquerdo em comparação com outras localizações cerebrais. ${ }^{1}$ A associação entre disfunção frontal e depressão é reforçada também por estudos 
controlados utilizando técnicas de neuroimagem estrutural em amostras de pacientes com transtornos depressivos maiores primários, que têm detectado alterações morfológicas especificamente em áreas de córtex pré-frontal nestes pacientes. ${ }^{11}$

É interessante notar que a paciente apresentava também história recente de alucinações visuais, cuja ocorrência é rara em síndromes depressivas. Por outro lado, alucinações visuais estão entre os sintomas mais freqüentemente associados a doenças orgânico-cerebrais. ${ }^{12}$ Em casos de lesões cerebrais focais, alucinações visuais são mais comuns em associação com comprometimento occipital ou de outras porções das vias visuais, e com lesões localizadas em córtex temporal ou parietal. ${ }^{13}$ Por outro lado, alucinações visuais ocorrem também como parte de distúrbios globais do funcionamento mental, em casos de rebaixamento do nível de consciência (delirium) por distúrbios metabólicos, aumento de pressão intracraniana ou outras causas orgânicas. ${ }^{13}$ Neste contexto, as alucinações visuais de surgimento recente na nossa paciente, somadas aos déficits cognitivos e ao achado de lentificação discreta do traçado eletroencefalográfico, poderiam refletir a instalação de um quadro de rebaixamento discreto do nível de consciência, em superposição ao quadro depressivo crônico.

Por fim, o presente caso levanta a questão do papel clínico das avaliações de neuroimagem em psiquiatria. À exceção dos casos de suspeita de demência, exames de neuroimagem não são comumente vistos como instrumentos do dia-a-dia da prática psiquiátrica, mas sim como recursos de pesquisa, cada vez mais utilizados na elucidação da fisiopatologia dos transtornos mentais. ${ }^{11,14}$ No entanto, no nosso caso, o exame de RM foi fundamental para trazer à luz a etiologia orgânica do quadro psiquiátrico da paciente, e guiar o estabelecimento da conduta apropriada. Diversos aspectos clínicos do caso sugeriam a necessidade de investigação de causa orgânica por exames de TC ou RM de crânio, por tratar-se de quadro psiquiátrico de curso crônico com deterioração progressiva, com sintomas cognitivos e alucinações visuais, na ausência de antecedentes pessoais psiquiátricos ou história familiar de transtornos mentais. ${ }^{2}$ Fica assim ressaltada a importância do uso de exames de neuroimagem estrutural em casos selecionados de apresentação psiquiátrica atípica. Já o exame de SPECT cerebral, ainda que não essencial para a elucidação diagnóstica do caso ou estabelecimento da conduta, foi útil para demonstrar alterações do funcionamento cerebral de extensão significativamente mais ampla do que os limites do tumor frontal.

Em conclusão, relatamos um caso psiquiátrico no qual a sintomatologia e curso do quadro levantavam a possibilidade de etiologia orgânica cerebral. A elucidação diagnóstica do caso, com a identificação de um meningeoma frontal, foi pos- sibilitada pelo uso de recurso de neuroimagem estrutural indicado com prontidão.

\section{Agradecimentos}

Agradecemos à Sra. Célia Maria Dias Cheda pela ajuda na preparação deste manuscrito. Geraldo Busatto Filho é financiado pela Fapesp (Processo 95/9446-1 - Jovens Pesquisadores em Centros Emergentes).

\section{Referências bibliográficas}

1. Robinson RG. Neuropsychiatric consequences of stroke. Annu Rev Med 1997;48:217-29.

2. Skuster DZ, Digre KB, Corbett JJ. Neurologic conditions presenting as psychiatric disorders. Psychiatr Clin North Am 1992;15:311-33.

3. Mega MS, Cummings JL. Frontal-subcortical circuits and neuropsychiatric disorders. J Neuropsychiatry Clin Neurosci 1994;6:358-70.

4. Cummings JL. The neuroanatomy of depression. J Clin Psychiatry 1993;54:14-20

5. Davison K. Schizophrenia-like psychoses associated with organic cerebral disorders: a review. Psychiatr Dev 1983;1:1-34.

6. American Psychiatric Association. Diagnostic and Statistical Manual of Mental Disorders. $4^{\text {th }}$ ed. (DSM-IV). Washington (DC): American Psychiatric Association; 1994.

7. Rauch SL, Renshaw PF. Clinical neuroimaging in psychiatry. Harvard Rev Psychiatry 1995;2:297-312.

8. Klotz M. Incidence of brain tumors in patients hospitalised for chronic mental disorders. Psychiatr Q 1957;31:669-80.

9. Galasko D, Kwo-On-Yuen PF, Thal L. Intracranial mass lesions associated with late-onset psychosis and depression. Psychiatr Clin North Am 1988;11:151-66.

10. Walker AE, Robins M, Weinfeld FD. Epidemiology of brain tumors: the national survey of intracranial neoplasms. Neurology 1985;35:219-26.

11. Soares JC, Mann JJ. The anatomy of mood disorders. Review of structural neuroimaging studies. Biol Psychiatr 1997;41:86-106.

12. David AS, Busatto GF. The hallucination: a disorder of brain and mind. In: Ron MA, David AS, editors. Disorders of brain and mind. Cambridge: Cambridge University Press; 1997. p. 336-62.

13. Taylor D, Lewis S. Delirium. J Neurol Neurosurg Psychiatry 1993; 56:742-51.

14. Busatto GF. SPET blood flow studies and the funcitonal anatomy of psychiatric disorders. In: Kerwin RW, editor. Neurobiology and Psychiatry vol 3 Neuroimaging in Psychiatry. Cambridge: Cambridge University Press; 1995. p. 81-109.

\section{Correspondência}

Geraldo Busatto Filho

Rua Ovídio Pires Campos s/n

CEP 05430-010

São Paulo, SP. Brasil. 